\title{
Importancia del mantenimiento en edificios escolares sismorresistentes en zona de licuación durante la pandemia.
}

\author{
N. Maldonado ${ }^{1 *}$, I. Maldonado ${ }^{1}$, A. Cueto ${ }^{1}$, P. Martín ${ }^{1}$, D. Buss ${ }^{1}$ \\ *Autor de Contacto: ngm@frm.utn.edu.ar \\ ${ }^{1}$ Centro Regional de Desarrollos Tecnológicos para la Construcción, Sismología e Ingeniería Sísmica \\ (CeReDeTeC), Facultad Regional Mendoza, Universidad Tecnológica Nacional, Mendoza, República Argentina
}

\section{RESUMEN}

El terremoto de San Juan, Argentina (19/01/2021) alertó sobre probables daños en escuelas en el Norte de Mendoza, Argentina, que es la zona de mayor peligro sísmico del país, por lo tanto, se realizó una inspección visual de daños en tres edificios educacionales en zona de riesgo potencial de licuación. Los daños observados en la mampostería encadenada debido al terremoto son leves pero los daños más significativos detectados encuentran su origen en los nueve meses de cierre de las escuelas, producto de la cuarentena impuesta a la educación, donde las condiciones ambientales como la presencia de napas freáticas superficiales impactan en el mantenimiento de las construcciones, requiriendo el control de funcionamiento de esos edificios aún durante este tipo de cierre.

Palabras clave: construcciones sismorresistentes; mampostería; napa freática; licuación; mantenimiento.

\section{Importance of Maintenance in Earthquake-Resistant School Buildings in the Liquefaction Zone During the Pandemic.}




\begin{abstract}
The earthquake in San Juan, Argentina (01/19/2021) warned of probable damage to schools in the North of Mendoza, Argentina, which is the area of greatest seismic risk in the country, therefore, a visual inspection of damage was carried out in three educational buildings in an area of potential risk of liquefaction. The damage observed in the tie-reinforced masonry due to the earthquake is slight but the most significant damage detected has its origin in the nine months that schools have been closed, as a result of the quarantine imposed on education, where environmental conditions such as the presence of surface water table has an impact on the maintenance of buildings, requiring control of the operation of these buildings even during this type of closure.
\end{abstract}

Keywords: earthquake resistant constructions; masonry; water table; liquefaction; maintenance.

\title{
Importância da manutenção em edifícios escolares resistentes a terremotos na zona de liquefação durante a pandemia.
}

\begin{abstract}
RESUMO
O terremoto em San Juan, Argentina (19/01/2021) alertou sobre prováveis danos a escolas no norte de Mendoza, Argentina, que é a área de maior perigo sísmico no país, portanto, uma inspeção visual dos danos foi realizado em três edifícios educacionais em uma área de risco potencial de liquefação. Os danos observados nas alvenarias acorrentadas devido ao terramoto são ligeiros mas os danos mais significativos detectados têm origem nos nove meses de encerramento das escolas, em resultado da quarentena imposta à educação, onde as condições ambientais como a presença de águas subterrâneas superficiais impactam a manutenção das edificações, exigindo o controle do funcionamento dessas edificações mesmo durante esse tipo de fechamento.
\end{abstract}

Palavras-chave: construções resistentes a terremoto; alvenaria; lençol freático; liquefação; manutenção.

\section{INTRODUCCIÓN}


La Organización para la Cooperación y el Desarrollo Económicos (OCDE, 2021) y la Organización de las Naciones Unidas para la Educación, la Ciencia y la Cultura (UNESCO) indican que el cierre de las escuelas durante la pandemia por la COVID-19 ubican a la Argentina entre los países que menos clases presenciales han tenido desde marzo de 2020 y consideran que la falta de infraestructura en las instituciones educativas fue el mayor obstáculo para continuar con las clases presenciales en toda la región debido a las salas de clase pequeñas, falta de baños y escuelas que no cumplen con los criterios para reabrir sus puertas en la pandemia.

La importancia de tener ambientes en buen estado aporta a que los estudiantes obtengan los resultados esperados en el proceso del aprendizaje, por lo que una buena infraestructura educativa conlleva a una mejora en la calidad educativa y logra una huella significativa sobre el rendimiento de los estudiantes (Cardini et al, 2020) ya que las escuelas inspeccionadas son las únicas escuelas de nivel inicial en cada distrito (POT Lavalle, 2019).

Si a la problemática de mantenimiento rutinario de edificios escolares se le incorpora el cierre por pandemia y la sismicidad local, el costo del mantenimiento de la infraestructura se incrementa.

Las escuelas inspeccionadas en febrero de 2021 se ubican en la zona de suelos rígidos profundos de acuerdo a la Microzonificación sísmica del Gran Mendoza (INPRES,1989), por lo que habitualmente el grado en la escala Modificada de Mercalli aumenta, cuando se origina un sismo de foco cercano. Los conos aluviales, cuando las pendientes naturales disminuyen, se encuentran cubiertos, en general por capas de suelos finos, en la mayoría de los casos con poca plasticidad, situación que genera problemáticas de baja capacidad portante por presencia de humedad y dificultades en el drenaje. Esta situación se presenta en la Figura 1 que indica la zona de llanura pluvio-aluvional y de transición del noreste mendocino.

El análisis geomorfológico del departamento de Lavalle, indica que el mismo se sitúa en la unidad morfo-estructural de las Planicies, formadas por depósitos continentales, predominantemente aluviales de origen terciario y cuaternario. El área comprendida por los distritos de Tulumaya, La Pega, Tres de Mayo, El Vergel, El Chilcal, Costa de Araujo y Gustavo André se desarrolla en la planicie aluvial con modificaciones antrópicas, donde los oasis son el lugar de asentamiento de la mayor parte de la población, sitio donde se desarrollan los cultivos y las áreas urbanas, formado por los abanicos aluviales producto del depósito de materiales arrastrados aguas abajo por el Río Mendoza. El área central del departamento, ocupado por la llanura oriental de origen fluvio - eólica ocupa la mayor parte de los distritos de La Asunción, San Miguel y Lagunas del Rosario (POT Lavalle, 2019).

Por la conformación de la planicie aluvial y el nivel de napa freática superficial, ante un terremoto significativo (M>7) es una zona de licuación potencial (CCSR, 1987). La Figura 2 presenta las isóbatas de la década anterior en la zona donde se ubican las escuelas inspeccionadas.

Los estudios de la licuación histórica en territorio argentino sostienen que la probabilidad de licuación disminuye mucho por debajo de los $3 \mathrm{~m}$ del nivel freático y las altas tasas de sedimentación favorecen el fenómeno (sedimentos de granos finos, lacustres y fluviales), aunque el terremoto de 1977 de San Juan ha detectado el fenómeno a más de 200 km del epicentro (Perucca et al. 2006). La explicación a esta situación es que los valles donde asientan las principales ciudades del oeste argentino se ubican sobre depósitos fluviales no consolidados, lugares donde no siempre se han realizado estudios de suelos previos a la construcción sismorresistente y que esto forma parte del riesgo al que están expuestas las edificaciones y las infraestructuras ante sismos significativos $(M>7)$. 


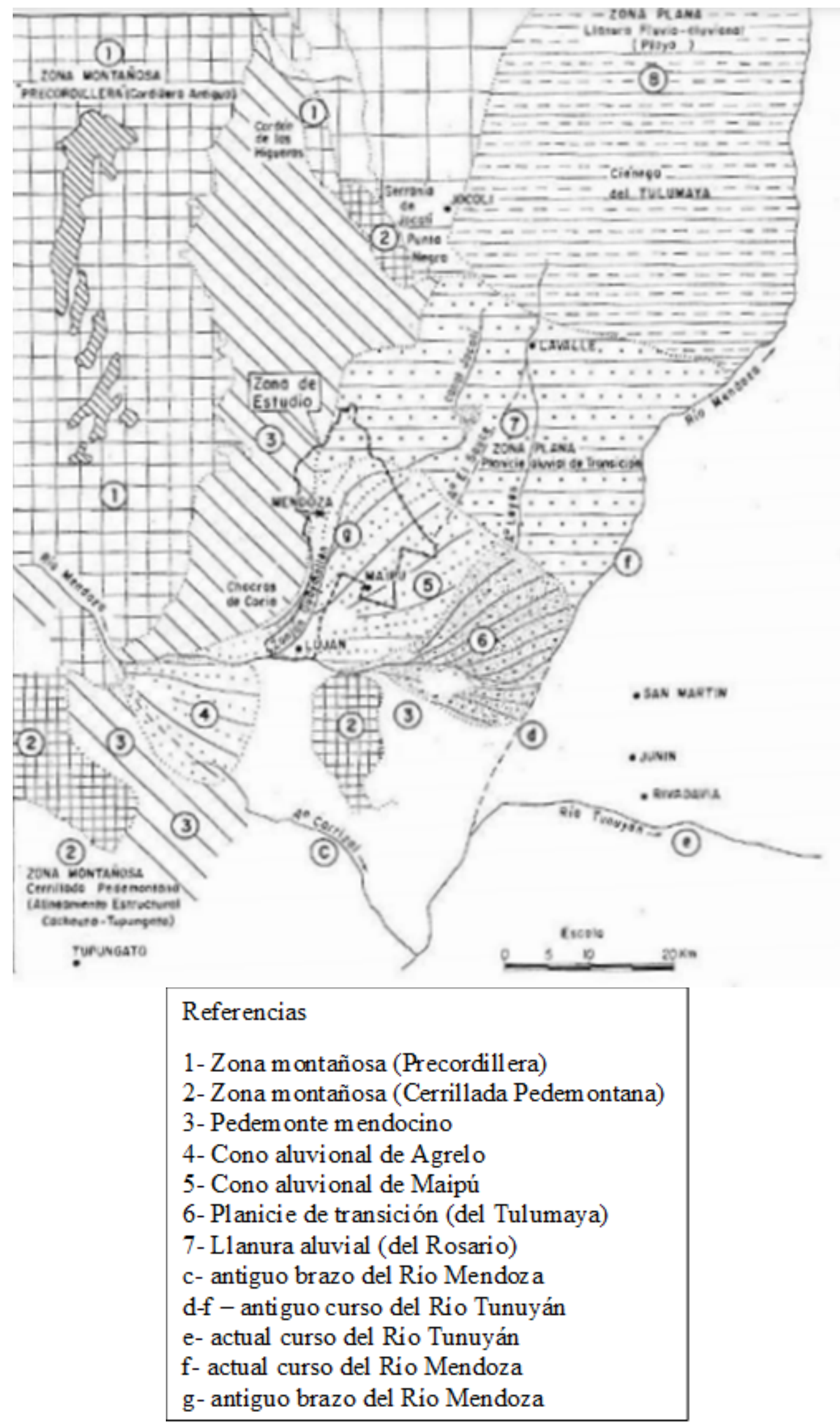

Figura 1. Unidades geológicas de Norte mendocino (Fuente: INPRES, 1989) 


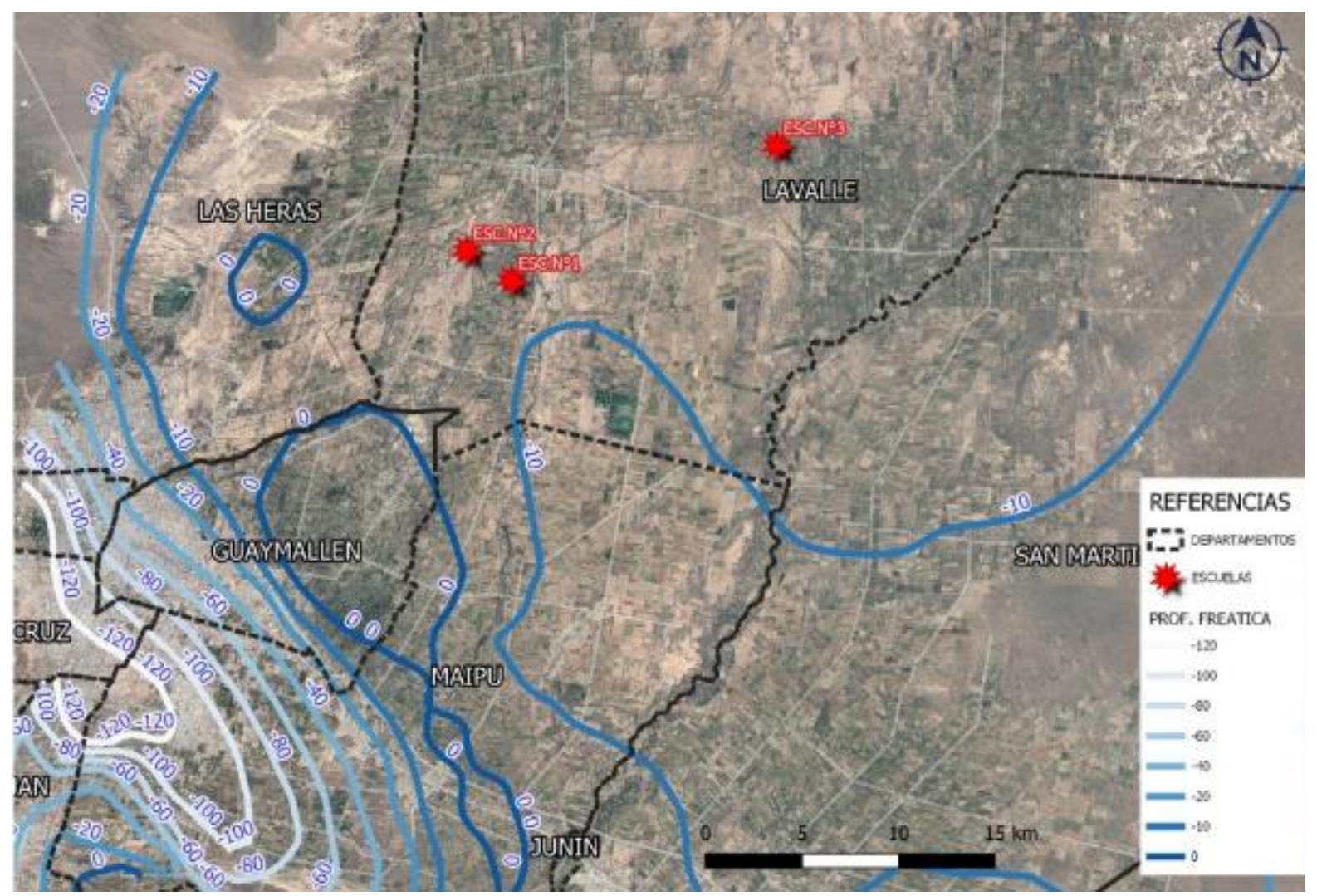

Figura 2. Curvas de igual nivel freático (Fuente: INA, 2011).

La licuación del suelo se produce cuando se rompe el equilibrio estático por aplicación de acciones estáticas o dinámicas en suelos con reducida resistencia residual. Las acciones externas derivan en un crecimiento de las presiones de poros, sin posibilidad de disipación rápida en función del tiempo de carga, produciendo grandes deformaciones y desplazamientos del suelo y afectando a las construcciones. Los factores que afectan el fenómeno son las condiciones de niveles de humedad del suelo, saturados o cercanos a la misma; la permeabilidad reducida que limita la disipación rápida; la resistencia movilizada por la fricción y el confinamiento reducido en relación con las solicitaciones actuantes (Maldonado et al, 2015).

Desde el punto de vista estructural, los edificios escolares evaluados están construidos con códigos anteriores a 1970 y no se encuentra documentación fidedigna del diseño estructural ni de las características de los materiales utilizados. La mampostería confinada es la modalidad más utilizada en esta región desde mitad del siglo XX con mampuestos cerámicos macizos artesanales con bordes encadenados de hormigón armado para mitigar los efectos de los asentamientos diferenciales en los muros (CCA, 1970; CCSR, 1987).

En cuanto a las fundaciones se estima que son de tipo superficial de hormigón armado y/o cimientos corridos y que posiblemente puedan estar apoyadas sobre algún suelo mejorado. Esta problemática de ausencia de historia documental de las escuelas se comparte con otros edificios escolares de Latinoamérica (INIFED, 2021).

\section{PROCEDIMIENTO}

Se procede a realizar una inspección visual acompañados por autoridades escolares ya que no cuentan con documentación del edificio, por lo que la antigüedad se estima en función de antecedentes aportados por la comunidad local, datos necesarios para iniciar la ficha de descripción y antecedentes de la estructura (CYTED, 1998). En esta inspección se identifican las principales 
patologías en relación con el terremoto más reciente y con las condiciones ambientales en relación a las condiciones de las fundaciones y las instalaciones. Finalmente se orientan a los directivos docentes sobre los pasos a seguir.

\section{RESULTADOS}

En la Tabla 1 se presentan los resultados de la inspección en base a las patologías detectadas.

Tabla 1: Resultados de las inspecciones oculares realizadas

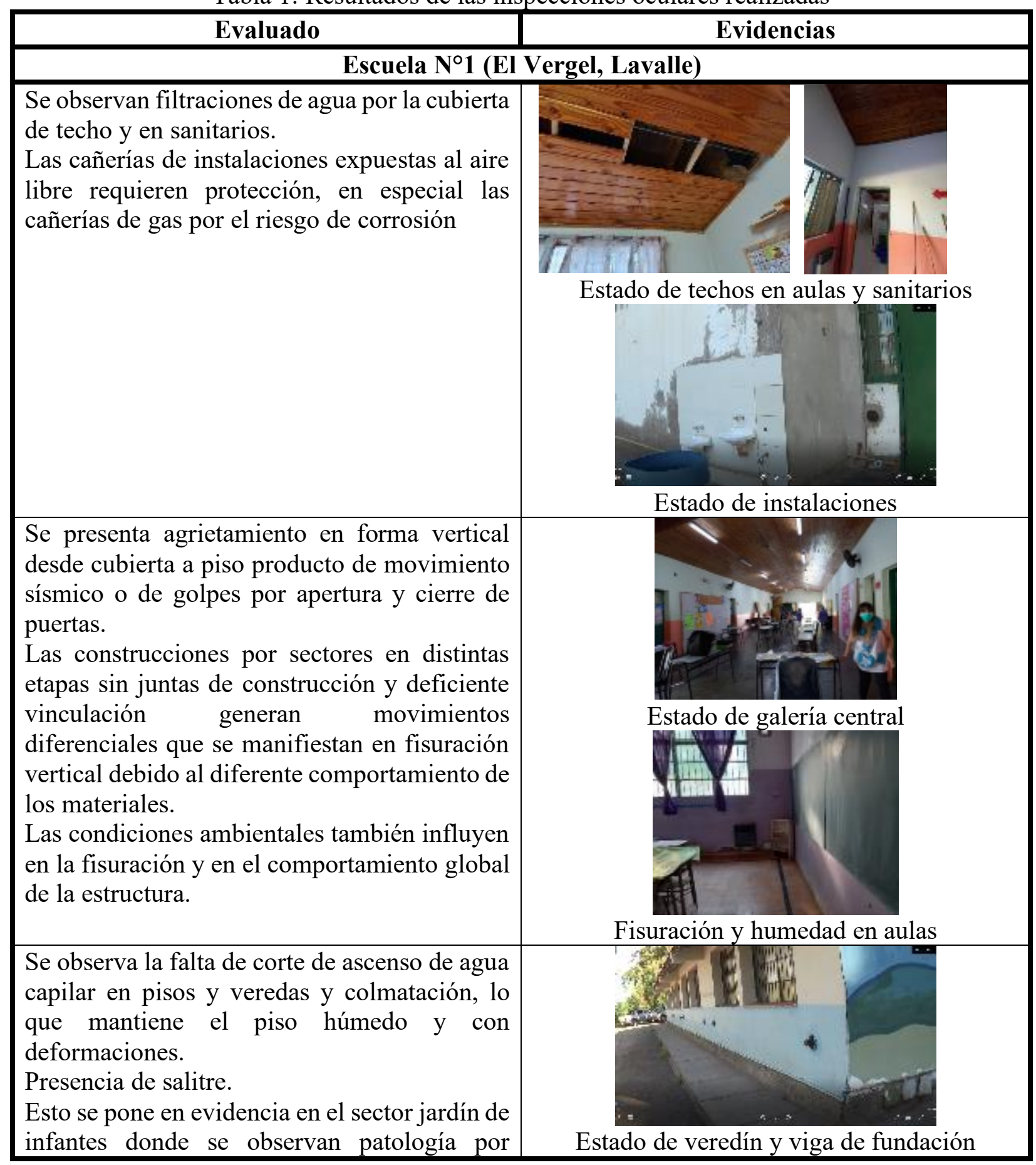




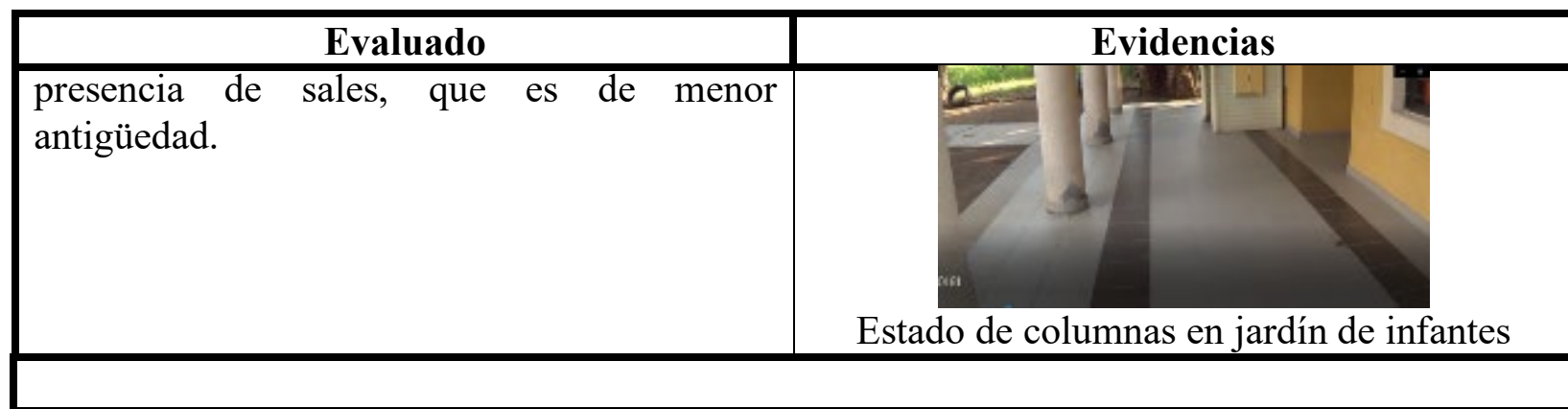

Se observan filtraciones de agua de la cubierta en distintas aulas, generando un serio problema de riesgo con las instalaciones eléctricas.

Las cañerías de instalaciones expuestas al aire libre requieren protección, en especial las cañerías de gas por el riesgo de corrosión.

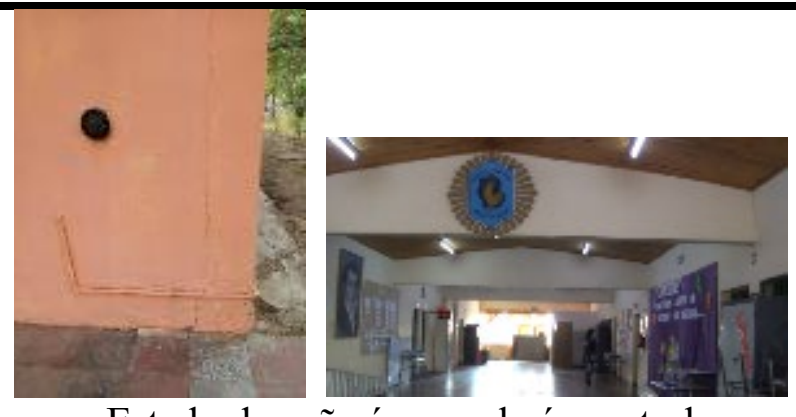

Se presentan agrietamientos en forma vertical desde cubierta a piso producto de movimiento sísmico o de golpes por apertura y cierre de puertas, que evidencian construcciones en diferentes etapas y comportamiento de materiales diferentes.

La fisuración puede estar relacionada con el comportamiento de los materiales, las condiciones ambientales y el comportamiento global de la estructura (cambios de rigidez en los elementos estructurales).

Se observa la falta de corte de ascenso de agua capilar en pisos y veredas lo que mantiene el piso húmedo $\mathrm{y}$ con deformaciones. La presencia de salitre y colmatación afecta revoques exteriores.

La estructura de vinculación inferior presenta salitre, lo que puede afectar su capacidad resistente por aumento de volumen $y$ disminución de secciones, aún en el sector más nuevo que es el jardín de infantes.

Estado de cañerías y galería central

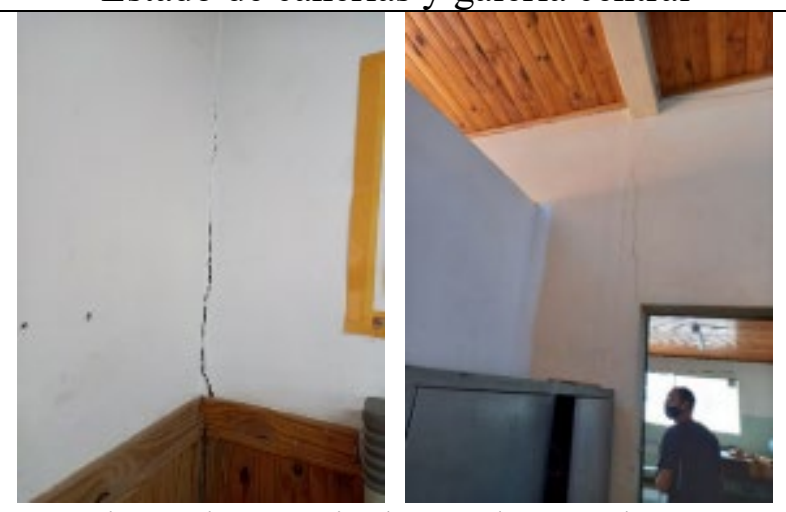

Fisuración vertical en aulas y galerías

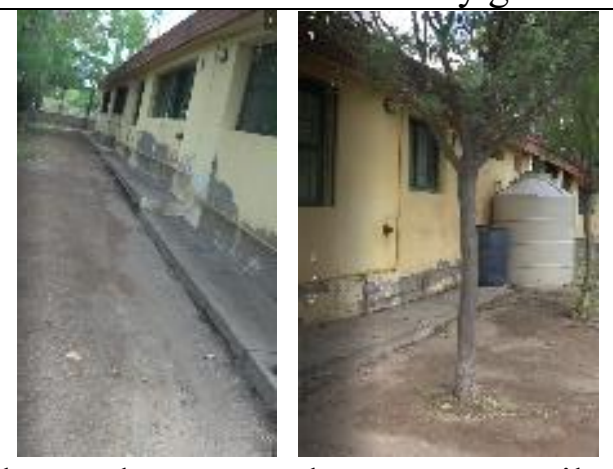

Problemas de ascenso de agua por capilaridad

\section{Escuela N`3 (El Plumero, Lavalle)}

Se observan filtraciones de agua en techos y en sanitarios.

Las cañerías de instalaciones expuestas al aire libre requieren protección, en especial las cañerías de gas por el riesgo de corrosión.

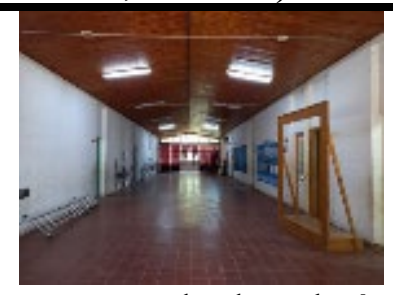

Estado de galería y aportes de agua 


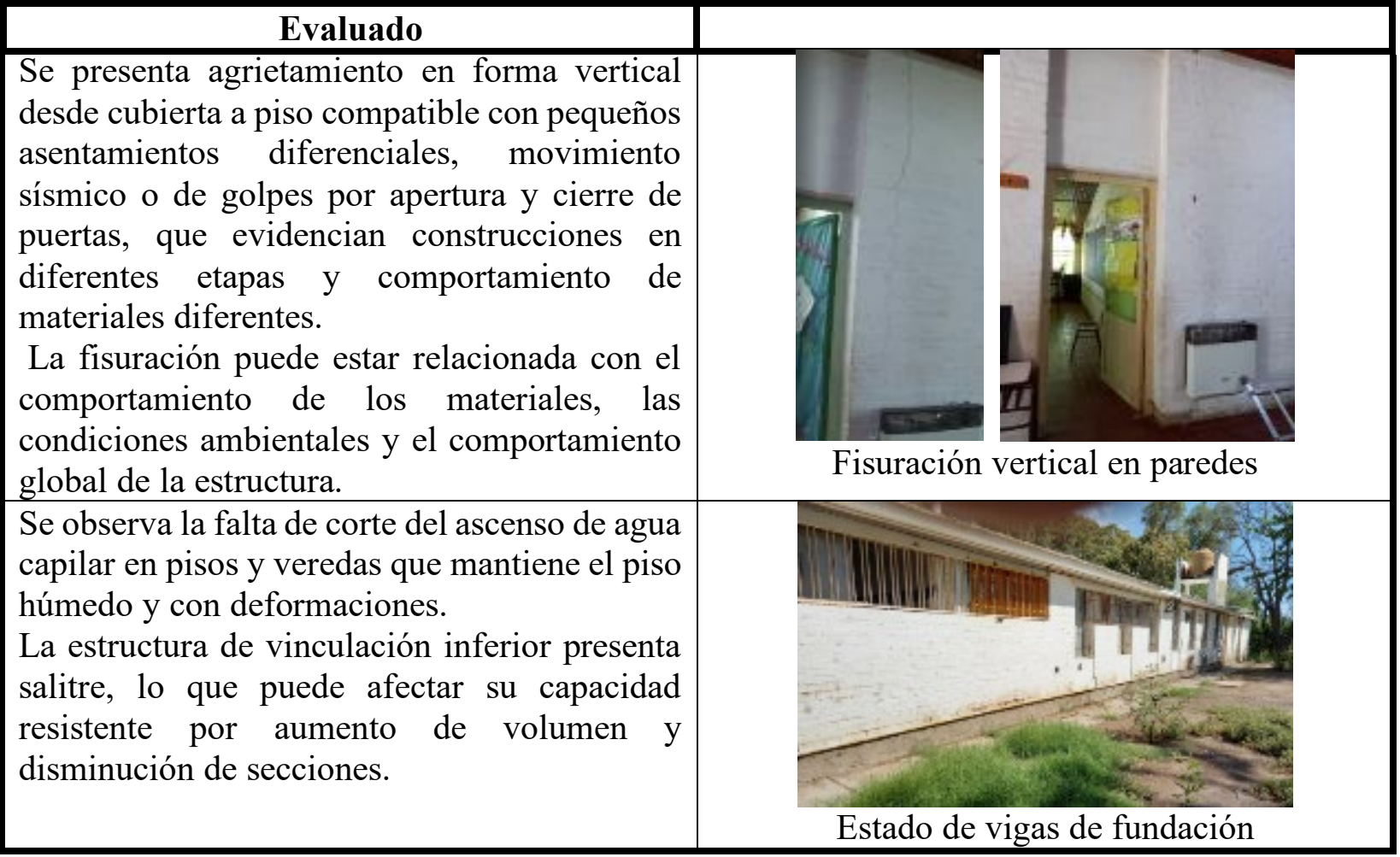

\subsection{Análisis de las inspecciones}

Las inspecciones realizadas ponen en evidencia la falta de documentación confiable del parque educativo provincial (Domizio et al, 2015) situación que a medida que transcurre el tiempo se acrecienta ya que las construcciones educativas también se van deteriorando.

Si bien la tecnología constructiva local se mantiene desde la década del 70 para estructuras sismorresistentes, los materiales locales utilizados han cambiado y no están presentando las mismas condiciones físico-mecánicas y de durabilidad de antaño (Maldonado et al, 1997), a lo que se suma el tema del mantenimiento del parque educativo y el aumento del vandalismo sobre la escuela.

Desde el punto de vista del comportamiento del suelo, a pesar de estar en zonas susceptibles a licuación, dicho fenómeno no se observa que haya afectado las estructuras hasta ahora. Sin embargo, se considera necesario actualizar las técnicas constructivas para la condición de cercanía de la napa freática, resolviendo las fundaciones con otras consideraciones que incluyan tecnologías que optimicen su durabilidad, en especial donde las condiciones de agresividad del agua que con el tiempo colmatan los capilares y modifican el comportamiento del terraplén de apoyo por saturación de la capa de corte de permeabilidad (Maldonado et al, 2015). Esto significa que el comportamiento de la fundación corrida presenta asentamientos diferenciales que afectan el comportamiento de toda la estructura superior y que las construcciones de mampostería evidencian muy rápidamente el problema mediante fisuración vertical como las observadas durante la inspección (CCSR, 1987).

Durante la cuarentena de la pandemia del año 2020 se mantuvieron cerradas las escuelas durante 9 meses hasta el punto que prácticamente no hubo mantenimiento de su infraestructura. En el caso de edificios escolares ubicados en zonas con cercanía a la napa freática, al cerrarse todas las aberturas se ha producido el aumento de la humedad ambiental interna afectando mampostería y terminaciones, que habitualmente una ventilación cruzada mantiene y atenúa el deterioro. La misma problemática de ausencia de mantenimiento ha incrementado los problemas de la evacuación de agua pluvial, afectando cubiertas y cielorrasos, generando problemas porque las aulas no pueden habilitarse por peligro de caída de cielorrasos y por ingreso de agua de lluvia. 
El tema de no deterioro de las instalaciones del edificio escolar es un tema recurrente y debiera implicar soluciones generales en el diseño del edificio. Los arreglos parciales generan pérdidas y aumentan la problemática de corrosión de cañerías por permanecer a la intemperie sin la protección adecuada, aumentando el riesgo de la población escolar y los costos de reparación.

\subsection{Continuidad de los estudios}

Con la información y documentación disponible no se puede realizar un diagnóstico acabado por lo tanto, se sugiere completarlo para cada escuela con las siguientes tareas:

1- Monitoreo de la fisuración en planos de planta y cortes, con colocación de testigos de vidrio de reloj que permitan identificar si la apertura de las mismas crece o no.

2- Búsqueda de la información técnica sobre la estructura y proceso constructivo de la misma, con detalle de fundaciones e identificación de etapas.

3- Estudio de suelos, de terraplenes, detección del nivel de la napa freática, identificación de sales expansivas e identificación de la estructura soporte.

4- Reparación de todas las pérdidas de agua y mantenimiento preventivo de todas las instalaciones de la escuela.

\section{CONCLUSIONES}

La inspección visual no es suficiente para aseverar que los daños detectados fueran causados por el terremoto más reciente, sin embargo, por las condiciones del lugar y tipo de suelos, existe una gran probabilidad de daño por ocurrencia del fenómeno de licuación de suelos. El nivel de fisuración detectado en las escuelas se considera leve (INIFED, 2021) aunque no se deba exclusivamente al terremoto de San Juan de enero de 2021.

Se considera de importancia incluir en el mantenimiento del parque escolar un tratamiento adecuado para las escuelas ubicadas en zonas con napa freática cercana, en especial en época de suspensión de actividades por grandes lapsos de tiempo en su uso.

Se debe implementar una adecuada recolección de información del parque escolar en lo que respecta a su estructura y a los cambios sufridos en el tiempo para evitar intervenciones de gran valor económico (INIFED, 2021).

\section{AGRADECIMIENTOS}

Este trabajo es parte de los Proyectos PID 4880TC y PID 5376 de la Universidad Tecnológica Nacional Facultad Regional Mendoza y PICT 2015-761 Agencia Nacional para la Promoción Científica y Tecnológica. Los autores desean agradecer a las instituciones que han permitido el desarrollo del trabajo.

\section{REFERENCIAS}

Alcocer Martínez de Castro S. et al. (2021). "Evaluación postsísmica de la infraestructura física educativa de México". Volumen 2: Introducción al comportamiento sísmico de estructuras para fines de evaluación. INIFED, México, p. 174.

Domizio M.C., Calderón F. y Maldonado N. (2015), Los riesgos de terremotos en construcciones escolares patrimoniales en zona de elevada sismicidad (Gran Mendoza). In Viand J. y Briones F., "Riesgos al sur. Diversidad de riesgos de desastres en Argentina”. La Red de Estudios Sociales en Prevención de Desastres en América Latina, cap. 12, pp. 177-196 
Cardini A., D'Alessandre V, Torre E. (2020). Educar en pandemia: respuestas provinciales al COVID. https://www.cippec.org/publicacion/politica-educativa-ante-el-covid-mendoza/ Acceso 05/05/2021.

CYTED Programa Iberoamericano de Ciencia y Tecnología para el Desarrollo (1998). "Manual de inspección, evaluación y diagnóstico de corrosión en estructuras de hormigón armado”. CYTED. Gobierno de Provincia de Mendoza (1970). Código de Construcciones Antisísmicas (CCA).

Gobierno de Provincia de Mendoza (1987). Código de Construcciones Sismorresistentes (CCSR). INA Informe IT No 124 - CRA (2011). Análisis de la evolución de las reservas hídricas subterráneas a base de las mediciones de las redes monitoras en las cuencas Norte y Centro de Mendoza.

INPRES. (1989). “Microzonificación Sísmica de Mendoza”. INPRES, San Juan, Argentina. Maldonado, I., Buss, D., Maldonado, N. (2015). "Importancia de los estudios del suelo para ubicar viviendas sociales en una zona sísmica licuable” in: Memorias XIII Congresso LatinoAmericano de Patologia da Construção, XV Congresso de Controlo de Qualidade na Construção, Congresso Luso-Africano da Construção, ALCONPAT Internacional, Lisboa: Portugal, Sesión 01paper 7208 fs. 8 .

Maldonado, I., Buss, D., Maldonado, N., Doña W. (2016). “Evaluación de terrenos con potencial de licuación en zona de alto riesgo sísmico: un caso de estudio" in: Memorias XXIII CAMSIG Congreso Argentino de Mecánica de Suelos e Ingeniería Geotécnica, SAIG, Santa Fe: Argentina, pp. 548-557

Maldonado,N., Michelini R., Olivencia L. (1997). "Análisis experimental de la degradación de mampostería encadenada para diseño estructural sismorresistente" in: Memorias XXVIII Jornadas Sudamericanas de Ingeniería Estructural, ASIE, San Carlos: Brasil, V.1 pp. 299-308. Municipalidad de Lavalle (2019). "Plan de Ordenamiento Territorial Municipal”. p.434.

OECD (2021), OECD Economic Outlook, May 2021, OECD Publishing. http:/www.oecd.org/economy/outlook/ acceso 31-05-2021

Perucca L., Pérez A. y Navarro C. (2006). Fenómenos de licuefacción asociados a terremotos históricos. Su análisis en la evaluación del peligro sísmico en la Argentina. Revista de la Asociación Geológica Argentina 61 (4) pp. 567-578 